\title{
Imprescriptibilidad y retroactividad de los Derechos y Garantías Constitucionales en la Constitución boliviana
}

\begin{abstract}
Non-applicability and retroactivity of Constitutional Rights and Guarantees in the Bolivian Constitution
\end{abstract}
Não aplicabilidade e retroatividade dos Direitos e Garantias Constitucionais na Constituição boliviana

Artículo recibido en enero 2021

Arbitraje en febrero 2021

Aceptación en marzo 2021

Publicación en abril 2021

\section{Flavio Abastoflor Dupleich}

fabastoflor6@gmail.com

https://orcid.org/ 0000-0001-6322-9572

Universidad Mayor Real y Pontificia de San Francisco Xavier de

Chuquisaca, Sucre, Bolivia
RESUMEN

ABSTRACT

RESUMO
La investigación tuvo como objetivo general proponer el diseño y la incorporación de un parágrafo quinto en el artículo 13 Título II, de la Constitución Política del Estado que establezca la imprescriptibilidad de vulneraciones a derechos y garantías constitucionales, así como la retroactividad. Su metodología fue de tipo observacional, descriptivo, corte transversal, con un enfoque mixto. Se utilizaron los métodos, históricológico, comparativo, modelación y causal. La encuesta se utilizó como técnica y el cuestionario como instrumento para la recolección de datos. Usando el método Delphy para la validación del instrumento. La población en estudio estuvo conformada por 570 profesionales afiliados al Colegio Departamental de Abogados, mientras que la muestra fue de 137 profesionales. Se obtuvo como resultado que aún se vulnera y violan los derechos constitucionales en Bolivia, por lo que con la implementación de barreras constitucionales claras y de manera taxativa no existirá interpretaciones erróneas o de conveniencia.

Palabras clave: Derechos constitucionales; garantías constitucionales; vulnerabilidad; legislación comparada

The general objective of the investigation was to propose the design and incorporation of a fifth paragraph in Article 13 Title II, of the Political Constitution of the State that establishes the imprescriptibility of violations of constitutional rights and guarantees, as well as retroactivity. Its methodology was observational, descriptive, cross-sectional, with a mixed approach. The historical-logical, comparative, modeling and causal methods were used. The survey was used as a technique and the questionnaire as an instrument for data collection. Using the Delphy method for instrument validation. The study population consisted of 570 professionals affiliated with the Departmental Bar Association, while the sample was 137 professionals. It was obtained as a result that constitutional rights are still violated and violated in Bolivia. Therefore, with the implementation of clear and restrictive constitutional barriers, there will be no erroneous or convenient interpretations.

Key words: Constitutional rights; constitutional guarantees; vulnerability; comparative legislation

O objetivo geral da investigação foi propor a formulação e incorporação de um parágrafo quinto no Artigo 13, Título II, da Constituição Política do Estado, que estabelece a imprescritibilidade das violações de direitos e garantias constitucionais, bem como a retroatividade. Sua metodologia foi observacional, descritiva, transversal, com abordagem mista. Foram utilizados os métodos histórico-lógico, comparativo, modelagem e causal. A pesquisa foi utilizada como técnica e o questionário como instrumento de coleta de dados. Usando o método Delphy para validação de instrumentos. A população do estudo foi composta por 570 profissionais vinculados à Ordem dos Advogados Departamentais, enquanto a amostra foi de 137 profissionais. Foi obtido como resultado que os direitos constitucionais ainda são violados e violados na Bolívia. Portanto, com a implementação de barreiras constitucionais claras e restritivas, não haverá interpretações errôneas ou convenientes.

Palavras-chave: Direitos constitucionais; garantias constitucionais; vulnerabilidade; legislação comparativa 
INTRODUCCIÓN

Las personas del siglo XXI transitan por diversos países cruzando fronteras por motivos laborales, turistas, exilios, estudios, deportes, entre otros. Cada acontecimiento de este tipo representa para el hombre el surgimiento de un acto jurídico, por ende, la aplicación de una norma jurídica en un sistema jurídico determinado, cabe destacar que, ciertos derechos pueden no tener siempre el mismo alcance.

A su vez, la sociedad en general, básicamente por regla debería saber por qué los derechos del que se hablan son importantes para su existencia, sin ellos no podrían desplazarse, ni estando en el mismo país, ni menos cuando se tenga que cruzar la frontera política del país al que se pertenece. Los derechos constitucionales que ofrece la Constitución en el sentido de que se cumplirán y respetarán los derechos que ella consagra, tanto a lo que se refiere al ejercicio de carácter privado como al de los de índole pública, si no fueran las garantías que establece la misma Constitución, respecto de un derecho fundamental como es la libertad de expresión, como tal derecho sería sólo un símbolo o tal vez un enunciado teórico alejado de toda realidad social y política del Estado socialmente constituido.

Es importante resaltar que en Bolivia ya desde la época colonial y también como estado independiente ha tenido como denominador común la sistemática y generalizada vulneración de derechos y garantías constitucionales como un mal congénito propio a su naturaleza misma, situación en la que se ha ido avanzando con el paso del tiempo empero esta no es una problemática que se haya superado.

De esta forma, el artículo 13, Parágrafo I. de Constitución Política de Estado (Bolivia), señala Barrera (2011, pp 44-45), que los derechos fundamentales del ser humano no se reivindican, únicamente deben ser protegidos o tutelados por el Estado en caso de ser vulnerados o estén en riesgo de ser vulnerados. El Parágrafo III. del artículo 14 del mismo texto constitucional platea que, es el Estado quien garantizará a toda la sociedad, el libre ejercicio de los derechos establecidos en la misma Constitución, leyes y tratados internacionales de derechos humanos

Además, en el Estado Plurinacional de Bolivia se debe tener clara la interpretación respecto a que los derechos constitucionales de las personas no prescriban en el tiempo, es decir que, aunque pase mucho tiempo de esa vulneración existe la seguridad jurídica que se llevara a cabo un debido proceso para poder dar una sanción efectiva basada en la ley a esa afectación a un derecho o garantía fundamental independientemente del tiempo transcurrido.

A su vez, no existe una seguridad interpretativa de los que vulneran los derechos constitucionales tengan una retroactividad para contar con un debido proceso, ya que debido a la inexistencia normativa de la retroactividad e imprescriptibilidad de la facultad de accionar cuando se vulnere algún derecho o garantía constitucional, se corre el riesgo de vulnerar derechos constitucionales que van en perjuicio de las personas. 
Sin duda alguna una de las principales causas para poder implementar la retroactividad y la imprescriptibilidad, es la continua violación de los derechos y garantías constitucionales en el Estado Plurinacional de Bolivia, donde se vive una inseguridad jurídica que campea en todo el territorio nacional, donde lo sufren no solo personas sino también, instituciones quienes ven con mucha preocupación que existan limites jurídicos para poder hacer valer la derechos que no se los pueden perder por ningún motivo.

En este sentido, surge la siguiente interrogante en la investigación; ¿Cómo se podrá garantizar el cumplimiento de los Derechos Fundamentales y Garantías constitucionales en la Constitución Política del Estado Plurinacional de Bolivia para evitar que sean vulneradas y puedan cumplir, proteger y precautelar los fines para los cuales fueron creados?

Partiendo de esa pregunta, el estudio tiene por objetivo principal, proponer el diseño y la incorporación de un parágrafo quinto en el artículo 13 Título II, de la Constitución Política del Estado que establezca la imprescriptibilidad de vulneraciones a derechos y garantías constitucionales, así como la retroactividad.

Aunado a ello, la investigación tiene una relevancia social, ya que la presente propuesta irá a llenar un gran vacío que se tiene en la Constitución Política del Estado, que va a asegurar respecto a ciertos acápites que deben ser mejoradas, para que las personas puedan estar seguros jurídicamente, además no se vulneran los derechos constitucionales.

Adicional, la investigación presenta como sustento teórico lo expuesto por Mesa; Gisbert y otros (2001), al señalar que, la Constitución Política del Estado es el principio del ordenamiento jurídico y expresa el grado de acuerdo al que hemos llegado para vivir pacíficamente aceptando normas comunes.

\section{Estado actual de los derechos y garantías constitucionales en el Estado Plurinacional de Bolivia}

Desde la promulgación de la Constitución Política del Estado en 2009, la situación de los derechos humanos en el país ha cambiado. Del artículo 13 al 107, la Constitución Política del Estado, incorpora una amplísima gama de derechos y garantías constitucionales, que la convierten en un ejemplo en el contexto internacional. En comparación con la Constitución anterior, la actual, es sustancialmente más amplia en el reconocimiento de derechos individuales y colectivos, visibilizando particularmente los derechos de los pueblos indígenas originarios campesinos en múltiples artículos, así como los derechos de las mujeres en 24 articulados para la igualdad de género, y el respeto a los principios de no discriminación y no violencia.

A su vez, se establece una gama amplia de derechos humanos, dando relevancia a los derechos colectivos y derechos de las naciones y pueblos indígenas originarios campesinos, en una estructura autonómica de la gestión pública. 
De forma transversal se introdujo la defensa de derechos por grupos que consideran a los derechos civiles y políticos y los derechos económicos, sociales y culturales. La Constitución también considera explícitamente los derechos de las mujeres. Además de resaltar el hecho que al reconocernos como Estado Plurinacional se asume una nueva conciencia de igualdad plena respecto a las Naciones y Pueblos Indígena Originario Campesino, están ejerciendo un sentido de justicia con los pueblos que sufrieron durante más de 500 años las formas más viles de discriminación, racismo y destrucción cultural.

En este sentido, se toma en cuenta las garantías constitucionales, el cual es definido por Palacios como escudos que se encuentran plasmados en la Constitución Política del Estado, que aseguran a los ciudadanos, el respeto a los derechos fundamentales que está proclama.

Dermizaky (2007) conceptualiza a las garantías como las seguridades jurídicas institucionales que la propia ley señala para hacer posible la vigencia de los derechos y libertades reconocidas u otorgadas.

Estas garantías constitucionales pueden ser de tipo normativo, jurisdiccionales e institucionales.

Aunado a ello, es necesario mencionar los principales organismos internacionales que son la base de los derechos humanos, entre ellos, se tiene a la Organización de las Naciones Unidas, Corte Interamericana de Derechos Humanos, Instituto Interamericano de Derechos Humanos, Corte Europea de Derechos Humanos, Corte Penal Internacional, entre otras.

\section{Principios de los Derechos Fundamentales en la Constitución Política del Estado}

Noguera (2010) afirma que los derechos fundamentales están compuestos por un elemento moral y otro de derecho positivo; la conjunción e integración de ambos elementos es imprescindible para la plena vigencia y ejercicio de los derechos fundamentales.

Bajo este componente, el Estado no solo debe reconocer jurídicamente a los derechos fundamentales, sino que debe establecer un sistema sancionatorio a las personas que vulneren ese nuevo orden jurídico de la dignidad humana.

\section{Vulneración de los Derechos y Garantías Constitucionales}

Defensores de los derechos humanos están de acuerdo en que, tras muchos años después de su publicación, la Declaración Universal de los Derechos Humanos es todavía más un sueño que una realidad. Existen violaciones de estos derechos en todas partes del mundo. Por ejemplo, en el Informe Mundial de Amnistía Internacional (2009), y de otras fuentes muestra que, a los individuos, se les tortura o se abusa de ellos en 81 países por lo menos, enfrentan juicios injustos en por lo menos 54 países, y se les restringen sus libertades de expresión en por lo menos 77 países. 
Cabe destacar, que a las mujeres y niños en particular se les margina de numerosas maneras, la prensa no es libre en diversos países y se calla a los disidentes, con demasiada frecuencia en forma permanente. Aunque se han logrado algunas ganancias en las últimas seis décadas, las violaciones de los derechos humanos siguen azotando a nuestro mundo actual.

Un ejemplo de la vulneración de los derechos, se encuentra presente en el artículo 3 de la Declaración Universal de los Derechos Humanos, el cual hace referencia al Derecho de vivir en libertad, donde todo individuo tiene derecho a la vida, a la libertad y a la seguridad personal. Sin embargo, en la realidad no se lleva a cabo ya que, se calcula que 6.500 personas murieron en 2007 en los conflictos armados en Afganistán, casi la mitad de las muertes fueron de civiles no combatientes a manos de insurgentes. También asesinaron a cientos de civiles en ataques suicidas de grupos armados. En Brasil, en 2007, según cifras oficiales, la policía mató por lo menos a 1.260 personas, la cifra más alta hasta la fecha. (Informe Mundial de Amnistía Internacional, 2009).

En este orden de ideas, los derechos más quebrantados en Bolivia, son el acceso a la justicia, la presunción de inocencia, el derecho al honor, el derecho a la defensa y al debido proceso.

\section{Organismos encargados de la Constitución en Bolivia}

El principal organismo encargado de la Constitución en Bolivia está conformado por los Jueces y Tribunales Ordinarios en el control, Jueces y Tribunales Ordinarios en la tutela de los derechos fundamentales como parte del control de la constitucionalidad.

Ahora bien, esa labor de control de constitucionalidad respecto al ejercicio de los derechos fundamentales y garantías constitucionales, está encomendada a los jueces y tribunales ordinarios por las normas de la Constitución y la propia Ley 1836 del Tribunal Constitucional.

En consecuencia, las autoridades o funcionarios responsables de haber restringido o suprimido un derecho fundamental o garantía constitucional no pueden ni deben objetar la jurisdicción y competencia de los jueces o tribunales, invocando tener un nivel jerárquico superior al Juez o a los titulares del tribunal; tampoco pueden invocar inmunidad o fuero alguno. (Rivera, 2009; p.2)

\section{Legislación comparada}

\section{Ecuador}

Para la investigación se tomó en cuenta los artículos 1, 3,6 y 11 de la Constitución Política de Ecuador; en ella se resalta que el Estado ejercerá de forma inmediata el derecho de repetición en contra de las personas responsables del daño producido, sin perjuicio de las responsabilidades civiles, penales y administrativas. 
Además, el Estado será responsable por detención arbitraria, error judicial, retardo injustificado o inadecuada administración de justicia, violación del derecho a la tutela judicial efectiva, y por las violaciones de los principios y reglas del debido proceso.

Cuando una sentencia condenatoria sea reformada o revocada, el Estado reparará a la persona que haya sufrido pena como resultado de tal sentencia y, declarada la responsabilidad por tales actos de servidoras o servidores públicos, administrativos o judiciales, se repetirá en contra de ellos.

En este sentido, en general las disposiciones para el ejercicio de los derechos son iguales o muy similares a los de la Constitución del Estado boliviano en cuando a que los derechos se pueden ejercer individual o colectivamente, en cuanto a que existe igualdad y no discriminación en el ejercicio de los derechos, en las características de ejercicios de los derechos como interdependientes inalienables.

\section{Colombia}

Se tomó en cuenta para este estudio los artículos 1, 2, 5,6, 13, 16, 29, 83, 85,89, 91, entre otros de la Constitución de la República de Colombia.

ElEstadocolombianosibientampoco recogepersela garantía normativa de la imprescriptibilidad y retroactividad para investigar y sancionar vulneraciones a derechos humanos (lo que vuelve a ratificar la novedad y necesidad de la propuesta en el contexto latinoamericano) también es cierto y evidente que incorpora otras figuras que busca que las lesiones a derechos fundamentales no queden en la impunidad como el deber de reparar vulneraciones a derechos fundamentales como un deber primordial del Estado, o la responsabilidad que tienen el Estado y las personas por vulneraciones a derechos fundamentales, la Constitución colombiana es muy insistente en este aspecto y se puede considerar a estas disposiciones como altamente positivas, en vista que son garantías normativas que buscan evitar la violación de derechos fundamentales.

También, la Constitución colombiana realiza una tutela muy completa al debido proceso en muchos componentes empero la única restricción temporal que pone como presupuesto de afectación al debido proceso, es que no se juzgue a nadie con una ley que no se encontraba vigente y es importante ver que la propuesta que ahora se plantea de ninguna manera supone una afectación de este tipo por no generar efectos de juzgamiento con leyes no vigentes y esto ratifica que la reforma ahora propuesta no vulnerara el debido proceso.

Cabe destacar que las constituciones de Ecuador y Colombia contienen un mayor número de disposiciones e institutos jurídicos especializados para garantizar el ejercicio efectivo de los derechos humanos y reducir los índices de vulneración a los mismos.

Mientras que, la Constitución boliviana respecto a las disposiciones generales para la protección de los derechos tiene una gran debilidad y un gran retraso la Constitución de Colombia y 
fundamentalmente la de Ecuador es mucho más completa en ese tema, lo que demuestra que se debe progresivamente y a un ritmo acelerado incorporar otras normas para la defensa y vigencia efectiva de los derechos humanos.

MÉTODO

El estudio se desarrolló con una metodología de tipo observacional, descriptivo, porque permitió disponer de un panorama más preciso de la magnitud del problema, se evidenciaron situaciones y eventos, se buscó especificar las propiedades importantes del tema de investigación. Se midieron y evaluaron diversos aspectos, dimensiones o componentes, del fenómeno investigado, jerarquizando sus componentes para estructurar estrategias operativas.

De corte transversal, porque midió el comportamiento del fenómeno investigado, tal como se presentó en el momento de estudio. Con un enfoque mixto, cuali-cuantitativo, por el uso de herramientas cualitativas y la ayuda de métodos cuantitativos, para la aplicación del instrumento y el posterior análisis teórico de dichos resultados, en la misma se recogió, observó y analizó datos cuantitativos medibles, verificables, comparables como auxiliares para el diagnóstico del tema de estudio, sin dejar de tener algunos rasgos dialécticos de tipo cualitativo que permitieron la realización del mismo.

Los métodos que se utilizaron durante el desarrollo teórico de la investigación fueron históricológico, comparativo, modelación y causal.

Histórico-lógico. A través de este método se estudió el impacto evolutivo de la atención con calidad a los asegurados para responder al encargo social.

Comparativo. Se utilizó con el fin de obtener, organizar y sistematizar los datos disponibles, sometiéndolos a un conjunto de operaciones y comparación con vistas a utilizarlos como fuente de información, permitiendo el análisis de todos los documentos de investigación.

Modelación. Se utilizó en la estructuración teórica tanto en los procesos del marco teórico como de la propuesta final, permitiendo descubrir y estudiar nuevas relaciones y cualidades entre el respeto a los derechos constitucionales y la retroactividad e imprescriptibilidad de los mismos.

Causal. Se aplicó este método debido a que se pudo identificar las posibles causas de una existencia o no de una correcta vulneración de derechos constitucionales por personas e instituciones en el Estado Plurinacional de Bolivia.

Continuando con la metodología de la investigación, se utilizó para la recolección de datos como técnicas e instrumentos, la encuesta y entrevista. Sin embargo, para el estudio solo se tomó en cuenta los resultados obtenidos por la encuesta. 
Encuesta. Para este estudio científico se utilizó la encuesta con su herramienta el cuestionario, lo que permitió un análisis dialectico cuali-cuantitativo, porque se percibió directamente el objeto de investigación, el cual fue el proceso de cumplimiento de los derechos y garantías constitucionales en el Estado plurinacional de Bolivia respecto a la irretroactividad e imprescriptibilidad como alternativas jurídicas de solución y mecanismos extras de tutela judicial.

Cuestionario. Como instrumento de la encuesta, se utilizó para realizar el contraste de la encuesta realizada a los asegurados del Colegio Departamental de Abogados, donde se emplearon los mismos parámetros en la elaboración del cuestionario con preguntas cerradas.

Entrevista. Este instrumento se utilizó para recoger información directa sobre los informantes claves (personas con todo el conocimiento técnico científico suficiente y comprobado para poder responder a las interrogantes que se necesitan para la elaboración de la investigación), se utilizó la guía de entrevistas las cuales fueron lo más lógicas y coherentes para buscar la fundamentación de lo investigado.

El método Delphy fue utilizado para la validación de los instrumentos, este método se realizó a través de un cuestionario, el cual fue enviado a expertos, para que los mismos analicen la pertinencia del diseño y la implementación de un parágrafo en el artículo 13 Título II, Derechos Fundamentales y Garantías, Capitulo Primero de la Constitución Política del Estado Plurinacional de Bolivia. La información obtenida fue procesada estadísticamente.

Cabe destacar, que la información se acopió mediante las técnicas de revisión documental para conocer los fundamentos del problema fue diseñado tres cuestionarios de acuerdo a la población de estudio, el cuestionario se estructuro de acuerdo al problema de la investigación, de donde salieron las variables o ítems para realizar la encuesta. El cuestionario fue elaborado en escalas de acumulación tipo Likert.

Por otro lado, la población y muestra en estudio, estuvo conformada por 570 profesionales afiliados al Colegio Departamental de Abogados, mientras que la muestra fue de 137 profesionales.

Los criterios que se tomaron en cuenta para la inclusión de la población y muestra fueron: estar afiliado al Ilustre Colegio Departamental de Abogados, afiliados que asisten a las reuniones ordinarias del Ilustre Colegio Departamental de Abogados, afiliados con cuentas al día y no se discrimina el sexo, ni la edad.

RESULTADOS

Para este estudio solo se tomó en cuenta el instrumento encuesta, la misma tuvo como objetivo obtener información de los afiliados al ilustre Colegio de abogados de la ciudad de Sucre, 
quienes de manera directa fueron los que brindaron la información pertinente y aplicable respecto al diseño e implementación de la retroactividad y la imprescriptibilidad de los derechos constitucionales mediante la creación de un parágrafo quinto en el artículo 13 en el Titulo II, Derechos Fundamentales y Garantías, Capitulo Primero de la Constitución política del Estado Boliviano.

El diseño del cuestionario se realizó con la escala tipo Likert, que constó de 10 preguntas divididas en dos dimensiones (vulneración de los derechos constitucionales y propuesta de implementación de la retroactividad e imprescriptibilidad) con los valores correspondientes:

(1) Totalmente en desacuerdo.

(2) En desacuerdo.

(3) Ni de acuerdo ni en desacuerdo.

(4) De acuerdo.

(5) Totalmente de acuerdo.

\section{Primera dimensión. Vulneración de los Derechos Constitucionales}

En esta dimensión se tomaron en cuenta 5 ítems, los cuales fueron dirigidos a los profesionales afiliados al colegio de abogado de la ciudad de Sucre. En la Tabla 1, se aprecian los resultados obtenidos, tomando en cuenta la escala tipo Likert.

Tabla 1. Vulneración de los derechos constitucionales.

\begin{tabular}{|c|c|c|c|c|c|c|c|}
\hline \multirow{2}{*}{ Ítems } & \multicolumn{5}{|c|}{ Alternativas } & \multirow{2}{*}{\multicolumn{2}{|c|}{ TOTAL }} \\
\hline & 1 & 2 & 3 & 4 & 5 & & \\
\hline \multirow{2}{*}{ 1. Formación profesional } & 0 & 0 & 0 & 12 & 125 & 137 & Frecuencia \\
\hline & 0 & 0 & 0 & 9 & 91 & 100 & $\%$ \\
\hline \multirow{2}{*}{ 2. Aceptación } & 129 & 8 & 0 & 0 & 0 & 137 & Frecuencia \\
\hline & 94 & 6 & 0 & 0 & 0 & 100 & $\%$ \\
\hline \multirow{2}{*}{ 3. Divulgación } & 0 & 0 & 0 & 3 & 134 & 137 & Frecuencia \\
\hline & 0 & 0 & 0 & 2 & 98 & 100 & $\%$ \\
\hline \multirow{2}{*}{ 4. Sanciones } & 0 & 0 & 7 & 29 & 101 & 137 & Frecuencia \\
\hline & 0 & 0 & 5 & 21 & 74 & 100 & $\%$ \\
\hline \multirow{2}{*}{ 5. Intransferencia } & 0 & 0 & 0 & 0 & 137 & 137 & Frecuencia \\
\hline & 0 & 0 & 0 & 0 & 100 & 100 & $\%$ \\
\hline
\end{tabular}


La Tabla 1 muestra los resultados obtenidos de cada uno de los ítems que conforman la primera dimensión, en este sentido, en el primer ítem, sobre la enseñanza de los derechos constitucionales en la formación pregradual y posgradual se observa que un 91\% se identificó con la opción 5 es decir totalmente de acuerdo, y un $9 \%$ con la opción 4 de acuerdo, es decir que la gran mayoría están muy de acuerdo con la enseñanza de los derechos y garantías constitucionales.

En el segundo ítem muestra que un $94 \%$ de los encuestados, respondió con la opción 1, es decir, están totalmente en desacuerdo con que se violen derechos constitucionales, y solo un $6 \%$ con la opción 2 en desacuerdo que se sigan vulnerando los derechos y garantías constitucionales.

El tercer ítem, muestra que un $98 \%$ de los encuestados se identifica con la opción 5 , es decir, están totalmente de acuerdo, a que sí debería socializarse los derechos y garantías constitucionales en la población, mientras que solo un $2 \%$ de la población encuestada, prefiere la opción 4 estando de acuerdo con la socialización.

El cuarto ítem hace referencia sobre las sesiones a los vulneradores de derechos y garantías constitucionales, así que un $74 \%$ de los encuestados se identifican con la opción 5 , es decir, que están totalmente de acuerdo con el castigo, un 21\% con la opción 4 es decir de acuerdo con los castigos, y solo un 5\% con la opción 3 ni de acuerdo ni en desacuerdo.

En lo que concierne al quinto ítems, respecto a que los derechos constitucionales son inalienables, un $100 \%$ de todos los encuestados mencionó que sí están totalmente de acuerdo que no se los puede perder bajo ninguna consigna.

De esta manera, se evidenció que la gran mayoría de los encuestados estuvieron de acuerdo que aún se vulnera y violan los derechos constitucionales en Bolivia, y que además es importante hacer mención que los derechos y garantías constitucionales no prescriben tempero espacialmente.

\section{Segunda dimensión. Retroactividad e imprescriptibilidad}

Continuando con la segunda dimensión, se puede apreciar en la Tabla 2, en el sexto ítem que de todos los encuestados, un $82 \%$ considera que está totalmente de acuerdo con ponerle un límite a los derechos constitucionales, un $5 \%$ está de acuerdo, y por último un $20 \%$ de todos los encuestados ni de acuerdo ni en desacuerdo.

Tabla 2. Implementación de la retroactividad e imprescriptibilidad de los derechos constitucionales en el artículo 13.

\begin{tabular}{lccccccc}
\hline \multicolumn{1}{c}{ ítems } & $\mathbf{1}$ & $\mathbf{2}$ & $\mathbf{3}$ & $\mathbf{4}$ & $\mathbf{5}$ & \multicolumn{2}{c}{ TOTAL } \\
\hline 6. Mecanismo de tutela & 0 & 0 & 0 & 12 & 125 & 137 & Frecuencia \\
& 0 & 0 & 0 & 9 & 91 & 100 & $\%$ \\
7. Retroactivos e & 129 & 8 & 0 & 0 & 0 & 137 & Frecuencia \\
imprescriptibles & 94 & 6 & 0 & 0 & 0 & 100 & $\%$ \\
\hline
\end{tabular}




\begin{tabular}{lcccccccc}
\hline \multicolumn{1}{c}{ ítems } & \multicolumn{9}{c}{ Alternativas } & \multicolumn{2}{c}{ TOTAL } \\
& $\mathbf{1}$ & $\mathbf{2}$ & $\mathbf{3}$ & $\mathbf{4}$ & $\mathbf{5}$ & & \\
& 0 & 0 & 0 & 3 & 134 & 137 & Frecuencia \\
8. Diseño de propuesta & 0 & 0 & 0 & 2 & 98 & 100 & $\%$ \\
\hline $\begin{array}{l}\text { 9. Implementación de la } \\
\text { propuesta }\end{array}$ & 0 & 0 & 7 & 29 & 101 & 137 & Frecuencia \\
$\begin{array}{l}\text { 10. Beneficios de la } \\
\text { propuesta }\end{array}$ & 0 & 0 & 5 & 21 & 74 & 100 & $\%$ \\
\hline
\end{tabular}

En lo que respecta a que los derechos constitucionales sean retroactivos e imprescriptibles (séptimo ítem), un $97 \%$ del total de los encuestados está totalmente de acuerdo que lo sea, frente a un 3\% está de acuerdo a que los derechos y garantías no se pierdan en el tiempo ni en el espacio.

Además, en el octavo ítem referente a la propuesta el $99 \%$ de los encuestados está totalmente de acuerdo, y un $1 \%$ está de acuerdo con la propuesta de implementación y creación de la retroactividad e imprescriptibilidad en el artículo 13 de la Constitución Política del Estado.

Mientras que en el noveno ítem, un $99 \%$ de todos los encuestados se identifica con la opción 5 , es decir, están totalmente de acuerdo con la implementación de la propuesta, un $1 \%$ está identificado con la opción 4 es decir de acuerdo con la propuesta.

De todos los encuestados un $89 \%$ está identificado con la opción 5 es decir totalmente de acuerdo que la propuesta frenará la vulneración de los derechos constitucionales (décimo ítem), un $9 \%$ con la opción 4, es decir está de acuerdo que si se pondrá un límite a la violación de derechos constitucionales. Y por último, un $2 \%$ no está ni de acuerdo ni en desacuerdo.

De esta forma, se evidenció que en lo que concierte al acápite de la propuesta de crear e implementar la retroactividad y la imprescriptibilidad de los derechos constitucionales en el artículo 13 de la Constitución Política del Estado Boliviano, una gran mayoría estuvo de acuerdo, ya que consideró que es una limitante para que no se sigan violando y vulnerando los derechos de las personas. 


\section{Propuesta Incorporación del Parágrafo Quinto en el artículo 13 Título II, Derechos Fundamentales y Garantías, Capitulo Primero de la Constitución Política del Estado Plurinacional de Bolivia que establezca la imprescriptibilidad y retroactividad de vulneraciones a derechos fundamentales}

\section{Fundamentación de la propuesta}

Después de haber realizado el análisis, interpretación y proyección de las herramientas teóricas y empíricas las cuales posibilitaron estructurar la presente propuesta, se realizó un análisis constitucional para poder presentar una oferta real, aplicable y sobre todo pertinente a la realidad nacional.

\section{Método teórico}

Los métodos teóricos posibilitaron estructurar los lineamientos específicos de la propuesta, los cuales enmarcaron los aspectos técnicos y conceptuales para la implementación de la retroactividad e imprescriptibilidad de los derechos y garantías constitucionales en el texto constitucional boliviano.

\section{Métodos empíricos}

Los métodos empíricos con las dos herramientas utilizadas en la investigación dieron un resultado de la necesidad de garantizar la implementación de la retroactividad y la imprescriptibilidad de los de derechos y garantías constitucionales en el mismo texto constitucional, toda la información recogida permitió la realización de la propuesta, para de esta manera poder responder al problema de investigación central y cerrar el ciclo epistemológico de la investigación.

Por lo tanto, la fundamentación de la propuesta está dada por el análisis dialectico de las dos herramientas que se usaron para identificar los caminos y para poder cerrar- responder el problema de la investigación. De esta manera la presente investigación no solo se convierte en un aporte a la sociedad constitucional sino la sociedad en general.

\section{Objetivo de la propuesta}

Prevenir la violación de derechos y garantías constitucionales, sancionando las acciones y omisiones aplicando taxativamente el parágrafo quinto del artículo trece y de esta manera poder responder al encargo social.

\section{Justificación de la propuesta}

La propuesta está dividida en 3 áreas o pilares fundamentales: social, jurídico constitucional y político que buscan llenar un vacío existente hasta la actualidad para así responder al encargo social. 
Tabla 3. Propuesta Incorporación del Parágrafo Quinto en el artículo 13 Título II, Derechos Fundamentales y Garantías, Capitulo Primero de la Constitución Política del Estado Plurinacional de Bolivia que establezca la imprescriptibilidad y retroactividad de vulneraciones a derechos fundamentales.

\section{Artículo 13 antes de la propuesta}

\section{Artículo 13 con la propuesta}

\section{Artículo 13.}

I. Los derechos reconocidos por esta Constitución son inviolables, universales, interdependientes, indivisibles y progresivos. El Estado tiene el deber de promoverlos, protegerlos y respetarlos.

II. Los derechos que proclama esta Constitución no serán entendidos como negación de otros derechos no enunciados.

III. La clasificación de los derechos establecida en esta Constitución no determina jerarquía alguna ni superioridad de unos derechos sobre otros.

IV. Los tratados y convenios internacionales ratificados por la Asamblea Legislativa Plurinacional, que reconocen los derechos humanos y que prohíben su limitación en los Estados de Excepción prevalecen en el orden interno. Los derechos y deberes consagrados en esta Constitución se interpretarán de conformidad con los Tratados internacionales de derechos humanos ratificados por Bolivia.

\section{Artículo 13.}

I. Los derechos reconocidos por esta Constitución son inviolables, universales, interdependientes, indivisibles y progresivos. El Estado tiene el deber de promoverlos, protegerlos y respetarlos.

II. Los derechos que proclama esta Constitución no serán entendidos como negación de otros derechos no enunciados.

III. La clasificación de los derechos establecida en esta Constitución no determina jerarquía alguna ni superioridad de unos derechos sobre otros.

IV. Los tratados y convenios internacionales ratificados por la Asamblea Legislativa Plurinacional, que reconocen los derechos humanos y que prohíben su limitación en los Estados de Excepción prevalecen en el orden interno. Los derechos y deberes consagrados en esta Constitución se interpretarán de conformidad con los Tratados internacionales de derechos humanos ratificados por Bolivia.

V. Los derechos y garantías constitucionales insertas en el presente texto son de carácter imprescriptible y tienen retroactividad temporoespacialmente

\section{CONCLUSIONES}

Se ha llegado a la conclusión de la evolución de los derechos y garantías constitucionales en la realidad boliviana de donde se ha podido evidenciar que estos son de reciente aparición, y más nuevas aun en la Constitución Política del Estado, ya que, en los primeros textos no se contaba con el reconocimiento de los derechos humanos.

Todos los juristas, instituciones y teorías sobre los derechos y garantías constitucionales son de cumplimiento obligatorio por los Estados y gobernantes, en defensa de los ciudadanos, además de tener bien establecidos los mismos en organizaciones y en muchos textos como la declaración universal de los derechos humanos, insertas en la Constitución Política del Estado. 
Posterior a la aplicación del instrumento se puede decir que, 99\% de los encuestados están de acuerdo con la implementación del parágrafo $\mathrm{V}$ en el artículo 13 de la Constitución Política del Estado boliviano de la imprescriptibilidad y retroactividad de los derechos y garantías constitucionales, tal que, un $94 \%$ de los encuestados menciona que existe vulneración a los derechos y garantías constitucionales en Bolivia.

Para finalizar, la propuesta que se postula tiene pilares fundamentales y no solo se basa en los mismos, sino que se realiza una propuesta que busca la operatividad con la normativa legal vigente en el Estado Plurinacional de Bolivia, buscando entre las alternativas que permite la Constitución Política, misma propuesta que fue validada por los expertos en el área, para poder ser real, aplicable y sobre todo pertinente. Se llegó a la conclusión final que es necesaria la implementación de barreras constitucionales claras y de manera taxativa para que no existan interpretaciones erróneas o de conveniencia.

\section{REFERENCIAS}

Barrera, R. (2011). Derecho Constitucional. Montevideo: Editorial Rave

Constitución de la República de Ecuador. (2008). Constitución Política del Estado

Constitución Política del Estado Plurinacional de Bolivia (2009). Edición Oficial, La Paz, Gaceta Oficial de Bolivia

Constitución Poliúrica del Estado Colombiano Vigente. (2015) Recuperado en http:// www.corteconstitucional.gov.co/inicio/ Constitucion $\% 20$ politica $\% 20$ de $\% 20$ Colombia\%20-\%202015.pdf
Dermizaky, P. (2007). Derecho Constitucional. 4ta. Edición; Editorial J.V.; Cochabamba-Bolivia

Informe Amnistía Internacional (2009). El estado de derechos humanos en el mundo. Recuperado en https://www.amnesty.org/download/ Documents/48000/pol100012009spa.pdf

Mesa, J; Gisbert, T, y Mesa, C. (2001). Historia de Bolivia. Editorial Gisbert. La Paz-Bolivia

Noguera, A. (2010). Los derechos sociales en las nuevas constituciones latinoamericanas. Valencia, España: Editorial Tirant lo Blanch 